\title{
The effect of hormone therapy on all-cause and cardiovascular mortality in women with chronic kidney disease: protocol for a systematic review and meta-analysis
}

Sharanya Ramesh ${ }^{1,2}$, Michelle C Mann ${ }^{1,2}$, Jayna M Holroyd-Leduc ${ }^{1}$ Stephen B Wilton ${ }^{1,2}$, Matthew T James ${ }^{1,2,3}$, Ellen W Seely ${ }^{4}$ and Sofia B Ahmed ${ }^{1,2,3^{*}}$

\begin{abstract}
Background: Chronic kidney disease affects approximately one in ten North Americans and is associated with a high risk of cardiovascular disease. Chronic kidney disease in women is characterized by an abnormal sex hormone profile and low estradiol levels. Since low estradiol levels are associated with an increased cardiovascular risk in healthy women, our objective is to determine the effect of hormone therapy on all-cause mortality, cardiovascular mortality, and cardiovascular morbidity in women with chronic kidney disease.

Methods/design: Studies examining hormone therapy for adult women with chronic kidney disease will be included. The primary outcome is all-cause or cardiovascular mortality and morbidity. We will search electronic bibliographic databases (MEDLINE, EMBASE, and the Cochrane Central Register of Controlled Trials (CENTRAL)) along with relevant conference proceedings, table of contents of journals, and review articles. Two investigators will independently screen identified abstracts and select observational cohort studies, case-control studies, and randomized controlled trials examining hormone therapy in women with chronic kidney disease. These investigators will also independently abstract data from relevant full-text journal articles and assess risk of bias. Where possible, these data will be summarized using pooled or combined estimates for the risk ratio or hazard ratio of all-cause mortality, cardiovascular mortality, and cardiovascular morbidity in women with chronic kidney disease with and without hormone therapy. A random effects model will be used, and meta-regression and subgroup analyses will be used to explore potential source of heterogeneity.
\end{abstract}

Discussion: Given the high burden of cardiovascular disease in women with chronic kidney disease, this study will help guide clinical practice by summarizing current evidence on the use of hormone therapy for prevention of all-cause mortality, cardiovascular mortality, and cardiovascular morbidity in this population.

Systematic review registration: The final protocol was registered with PROSPERO (CRD42014014566).

Keywords: Hormone therapy, Chronic kidney disease, Cardiovascular mortality, All-cause mortality, Cardiovascular morbidity

\footnotetext{
* Correspondence: Sofia.Ahmed@albertahealthservices.ca

${ }^{1}$ Faculty of Medicine, University of Calgary, 3330 Hospital Drive NW, Calgary

T2N 4N1Alberta, Canada

${ }^{2}$ Libin Cardiovascular Institute of Alberta, 140329 Street NW, Calgary T2N

2T9Alberta, Canada

Full list of author information is available at the end of the article
}

C Biomed Central (c) 2015 Ramesh et al.; licensee BioMed Central. This is an Open Access article distributed under the terms of the Creative Commons Attribution License (http://creativecommons.org/licenses/by/4.0), which permits unrestricted use, distribution, and reproduction in any medium, provided the original work is properly credited. The Creative Commons Public Domain Dedication waiver (http://creativecommons.org/publicdomain/zero/1.0/) applies to the data made available in this article, unless otherwise stated. 


\section{Background}

Chronic kidney disease (CKD) is a prevalent condition with a high burden of all-cause and cardiovascular $(\mathrm{CV})$ morbidity and mortality [1,2]. Women with CKD have an abnormal sex hormone profile characterized by the lack of pulsatile gonadotropin-releasing hormone (GnRH) secretion and low estradiol levels $[3,4]$. As a low estrogen state is associated with increased CV risk [5], it may contribute to the increase in $\mathrm{CV}$ morbidity and mortality observed in women with CKD.

There is significant epidemiological evidence suggesting a cardioprotective function for ovarian sex hormones and, in particular, estradiol in women [6-12]. For example, the prevalence of cardiovascular disease (CVD) increases with age in men; however, women are relatively protected until menopause, after which time morbidity and mortality from CVD equal or exceed that of men [13]. Randomized control trials demonstrated no evidence of cardioprotection with hormone therapies [14-16]; however, later analysis of these studies have shown that the timing of hormone therapy initiation [17], route of administration of estrogen [18], and the type of estrogen and progesterone used [19] impact CV risk significantly.

In women with CKD, disruptions in the GnRH production lead to a hypoestrogenic state and cessation of menses and infertility, both of which return to normal levels with successful kidney transplants [20-23]. There is evidence to suggest that this loss of estradiol in women with CKD is associated with increased CV risk. In contrast to healthy women [24], women with end-stage kidney disease (ESKD) have similar overall mortality rates compared to age-matched men [25-27]. It is hypothesized that this increased risk may be due to the lower lifetime exposure to estradiol as a result of onset of kidney disease earlier in life [26]. In amenorrheic women with CKD, estrogen therapy has favorable effects on lipid profiles by decreasing LDL levels and increasing HDL levels [28-30].

Although a recent meta-analysis of postmenopausal hormone therapy in healthy postmenopausal women found no evidence of cardioprotection, the results varied among the included studies due to the different hormone regimens, the timing of hormone therapy initiation, and the type of estradiol and progestin used [19,31]. Additionally, it is important to note that 8 of the 13 trials included in the meta-analysis were examining the effect of hormone therapy as secondary, rather than primary, prevention in a high CV-risk population [31]. Whether these results are translatable to a high CV-risk population with CKD-mediated low levels of estradiol is unclear as the evidence has not been systematically summarized. Given the paucity of work on the therapeutic role of hormone therapy in the CKD population, we propose to complete a systematic review to determine the effect of hormone therapy on all-cause mortality, cardiovascular mortality, and cardiovascular morbidity in women with CKD. Additionally, given that the timing of the administration of hormone therapy (perimenopausal vs postmenopausal) influences risk, we aim to determine whether age, a surrogate for menopausal status, at administration of hormone treatment modifies outcomes in the CKD population [32].

\section{Methods}

\section{Design}

The systematic review protocol was designed based on the guidance from the Preferred Reporting Items for Systematic reviews and Meta-analyses Protocol (PRISMA-P) [33].

\section{Protocol}

To determine the effect of hormone therapy on allcause mortality, cardiovascular mortality, and cardiovascular morbidity (as defined by individual studies) in women with CKD, we developed a systematic review and meta-analysis protocol using the Cochrane guidelines for systematic review and meta-analysis [34]. The final protocol was registered with PROSPERO (CRD42014014566) [35]. The population, exposure, comparator, outcome, and study design (PECOD) utilized within this systematic review are presented in Table 1.

\section{Search strategy \\ Bibliographic databases}

The online databases to be searched are MEDLINE (1950 to September 2014), EMBASE (1980 to September 2014), and the Cochrane Central Register of Controlled Trials (CENTRAL). The Medline search will be peer reviewed [36].

\section{Other sources}

Two individuals will search the reference list of relevant publications, abstracts of all major North American Nephrology and Cardiovascular meetings, (American Society of Nephrology, Canadian Society of Nephrology, American Heart Association Scientific Sessions and American College of Cardiology) between 2011 and 2014, and the tables of contents of major nephrology journals (Journal of American Society of Nephrology, Kidney

\section{Table 1 PECOD question for the systematic review}

\begin{tabular}{ll}
\hline Population & $\begin{array}{l}\text { Women with chronic kidney disease (as defined by eGFR or } \\
\text { presence of kidney damage) }\end{array}$ \\
\cline { 2 - 2 } Exposure & $\begin{array}{l}\text { Hormone Therapy (HT) (all types of HT including } \\
\text { estradiol alone or estradiol and progesterone) }\end{array}$ \\
Comparator & Placebo or No HT \\
Outcome & All-cause and cardiovascular mortality \\
Design & RCTs, cohort, and case-control studies \\
\hline
\end{tabular}


International and American Journal of Kidney Diseases) and cardiology journals (Circulation, Journal of the American College of Cardiology) from January 2011 to September 2014. In order to get information about ongoing or unpublished studies, experts in the field will be contacted. Experts will be identified through the abstract review process.

\section{Search limits}

The search of online databases will include all languages and will not be restricted based on time period; however, it will be limited to studies on humans.

\section{Search terms}

Two separate searches will be conducted:

The first search will include the population and exposure terms of the PECOD question (Table 2):

\section{Chronic kidney disease}

The first Boolean search will be done using the "OR" term to combine "exploded" MeSH subject headings and "text word" searches of the following:

"Renal insufficiency chronic", "Kidney Failure",

"Kidney Diseases", "Renal Replacement Therapy",

"Uremia", "Dialysis", "Hemodialysis", "Hemofiltration",

"Peritoneal Dialysis", "Predialysis", "Kidney/Renal

Transplant", "Kidney/Renal Allograft", "End Stage

Renal/Kidney", "Chronic Kidney Failure" or "Chronic

Kidney Disease"

\section{Hormone therapy}

The second Boolean search will be done using the "OR" term to combine "exploded" $\mathrm{MeSH}$ subject headings and "text word" searches for the following:

"Hormone Therapy", "Hormone Replacement

Therapy", "Estrogen Replacement Therapy",

"Estrogen”, "Estradiol”, "Estriol”, “Conjugated Equine

Estrogen", "Progesterone", "Progestin",

"Medroxyprogesterone acetate" or "Dydrogesterone"

The above two will be combined using the "AND term.

The second search will include the exposure, outcome, and design terms of the PECOD question (Table 3). The purpose of this search is to identify randomized controlled trials that included subjects with CKD and conducted subgroup analysis for these subjects.

\section{Hormone therapy}

The first Boolean search will be done using the "OR" term to combine "exploded" MeSH subject headings and "text word" searches for the following:
"Hormone therapy", "Hormone Replacement

Therapy", "Estrogen Replacement Therapy",

"Estrogen", "Estradiol”, "Estriol”, "Conjugated Equine

Estrogen", "Progesterone", "Progestin",

"Medroxyprogesterone acetate", or "Dydrogesterone"

\section{Cardiovascular disease and all-cause mortality}

The second Boolean search will be done using the "OR" term to combine "exploded" MeSH subject headings and "text word" searches for the following:

"Cardiovascular Diseases", "Stroke”, "Hypertension”,

"Hyperlipidemias", "Arteriosclerosis", "Cholesterol”

"Blood Pressure", "Coronary Heart Disease",

"Mortality", "Survival Rate", "All Cause Mortality",

"Cause of Death", or "Death"

\section{Randomized control trial}

The third Boolean search will be done using the "OR" term to combine "exploded" MeSH subject headings and "text word" searches for the following:

\section{"Randomized Controlled Trial", "Controlled Clinical Trial", "Placebo", or "Drug Therapy"}

The above three will be combined using the "AND" term. Additionally, articles with the terms "Fabry's Disease" and "Growth Hormone Therapy" in the title or abstract will be excluded.

\section{Identification of articles for eligibility Initial screening}

After a calibration exercise in order to identify any systematic discrepancies in classification of abstracts between the two reviewers, screening will be conducted by two independent reviewers to determine articles eligible for the systematic review. An initial screen of identified abstracts from the databases and other sources will be performed to identify relevant articles for a full-text review. An abstract from the search will be considered for a full-text review if it meets the PECOD question criteria. This initial screening is intended to be sensitive and broadly capture any relevant articles studying hormone therapy in women with chronic kidney disease. In order to provide a comprehensive systematic review of the topic, the search and eligibility will not be restricted by a specific geographic region or publication year.

The decision of each reviewer for each abstract will be recorded, and any disagreement between reviewers will be resolved by consensus at all stages of the screening process. Authors will be contacted for missing data or clarification. For the initial screening search, if there is a lack of consensus among the reviewers and one of the 
Table 2 Search strategy for the chronic kidney disease and hormone replacement therapy search Population and Exposure terms of the PECOD question

Exp hormone replacement therapy/

2 Hormone replacement therap\$.tw.

3 (HRT or ERT or ORT).ti,ab.

4 Exp estrogen replacement therapy/

5 (Hormone adj3 replacement adj3 therap\$).mp.

6 (?Estrogen adj3 replacement adj3 therap\$).mp.

7 ?Estrogen replacement therap\$.tw.

8 ?Estradiol replacement therap\$.tw.

9 Hormone therap\$.tw.

10 Postmenopausal hormone therap\$.tw.

1 Post-menopausal hormone therap\$.tw.

121 or 2 or 3 or 4 or 5 or 6 or 7 or 8 or 9 or 10 or 11

13 Postmenopause.tw.

14 Exp postmenopause/

15 MENOPAUS\$4.tw.

6 POSTMENOPAUS\$4.tW.

7 POST-MENOPAUS\$4.tw.

8 Exp perimenopause/

9 Perimenopaus\$.tw.

Peri-menopaus\$.tw.

Exp menopause/

?Estrogen.tw.

Exp estrogens/

?Estradiol.tw.

Exp estradiol/

22 or 23 or 24 or 25

or/13-21

26 and 27

12 or 28

Exp kidney failure/

1 Exp chronic kidney failure/

2 Exp renal insufficiency/

3 Exp renal insufficiency, chronic/

4 Exp chronic kidney disease/

5 Exp kidney disease/

6 Exp renal replacement therapy/

7 Exp uremia/

38 Exp hemodialysis/

39 Exp kidney transplantation/

40 (Haemodialys* or hemodialys ${ }^{*}$ ).tw.

41 Dialysis.tw.

42 Peritoneal dialysis.tw.

43 Exp peritoneal dialysis/
Table 2 Search strategy for the chronic kidney disease and hormone replacement therapy search (Continued)

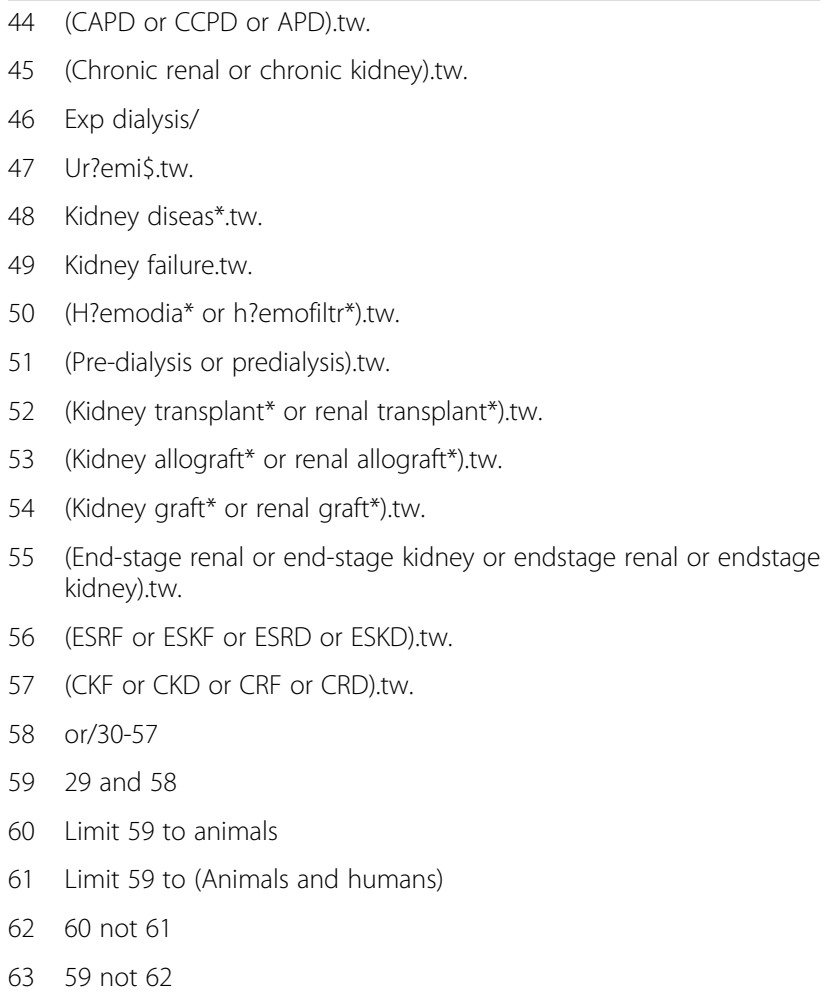

reviewers feels that a full-text review is required, the article in question will qualify for a full-text review.

\section{Full-text review}

The same two individuals will scan full-text articles, and those that meet the following criteria will be considered for the systematic review:

- Population: The study includes women with chronic kidney disease

- Exposure: Hormone therapy

- Comparator: Placebo or no hormone therapy

- Outcome: Hazards or risks of all-cause and cardiovascular mortality

- Study Design: RCT, cohort study, or case-control study

This will capture trials and cohort and case-control studies that compare hormone therapy to placebo or no hormone therapy on the risks or hazards of all-cause mortality, cardiovascular mortality, and cardiovascular morbidity in women with CKD for potential meta-analysis. The decision of each reviewer will be documented, and the agreement between the reviewers will be quantified by the Kappa statistic. Any disagreement between reviewers will be resolved by consensus. 
Table 3 Search strategy for the cardiovascular disease and hormone replacement therapy and randomized control trial search

\begin{tabular}{|c|c|}
\hline & Exposure, outcome, and design terms of the PECOD question \\
\hline 1 & Exp cardiovascular diseases/ \\
\hline 2 & Cardio*.tw. \\
\hline 3 & Cardia*.tw. \\
\hline 4 & Heart*.tw. \\
\hline 5 & Coronary.tw. \\
\hline 6 & Angina*.tw. \\
\hline 7 & Ventric .tw. \\
\hline 8 & Myocard*.tw. \\
\hline 9 & Pericard*.tw. \\
\hline 10 & Isch?em*.tw. \\
\hline 11 & Emboli*.tw. \\
\hline 12 & Arrhythmi*.tw. \\
\hline 13 & Thrombo*.tw. \\
\hline 14 & Atrial fibrillat*.tw. \\
\hline 15 & Tachycardi*.tw. \\
\hline 16 & Endocardi*.tw. \\
\hline 17 & (Sick adj sinus).tw. \\
\hline 18 & Exp Stroke/ \\
\hline 19 & (Stroke or strokes).tw. \\
\hline 20 & Cerebrovasc*.tw. \\
\hline 21 & Cerebral vascular.tw. \\
\hline 22 & Apoplexy.tw. \\
\hline 23 & (Brain adj2 accident*).tw. \\
\hline 24 & ((Brain* or cerebral or lacunar) adj2 infarct*).tw. \\
\hline 25 & Exp hypertension/ \\
\hline 26 & (Hypertension\$ or hypertensive\$).tw. \\
\hline 27 & Peripheral arter* disease ${ }^{*}$. tw. \\
\hline 28 & ((High or increased or elevated) adj2 blood pressure).tw. \\
\hline 29 & Exp hyperlipidemias/ \\
\hline 30 & Hyperlipid*.tw. \\
\hline 31 & Hypercholesterol*.tw. \\
\hline 32 & Hyperlipoprotein?emia*.tw. \\
\hline 33 & Hypertriglycerid?emia*.tw. \\
\hline 34 & Exp arteriosclerosis/ \\
\hline 35 & Exp cholesterol/ \\
\hline 36 & Cholesterol.tw. \\
\hline 37 & 'Coronary risk factor'.tw. \\
\hline 38 & Blood pressure/ \\
\hline 39 & Blood pressure.tw. \\
\hline 40 & or/1-39 \\
\hline 41 & Exp hormone replacement therapy/ \\
\hline 42 & Hormone replacement therap\$.tw. \\
\hline
\end{tabular}

Table 3 Search strategy for the cardiovascular disease and hormone replacement therapy and randomized control trial search (Continued)

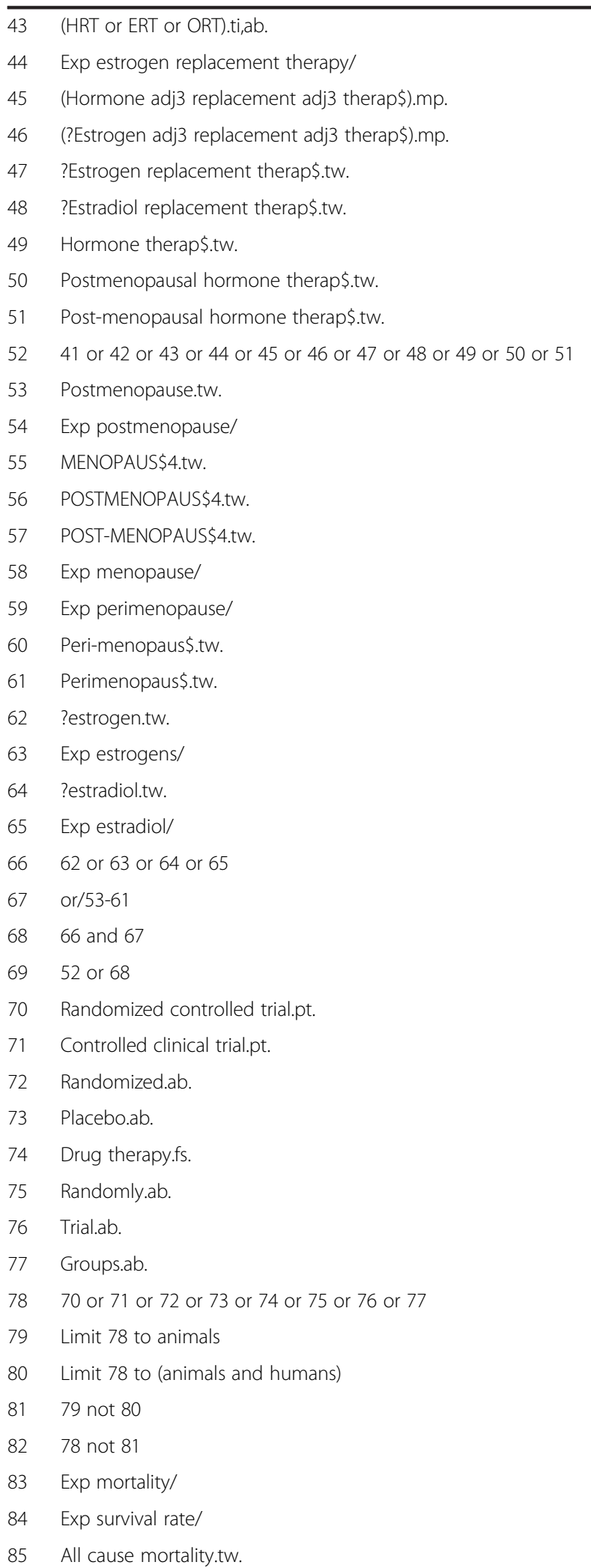




\section{Table 3 Search strategy for the cardiovascular disease and hormone replacement therapy and randomized control trial search (Continued)}

\begin{tabular}{ll}
\hline 86 & Mortality.tw. \\
87 & Cause of death.tw. \\
88 & Exp death/ \\
89 & Death.tw. \\
90 & Exp "Cause of death"/ \\
91 & Survival rate.tw. \\
92 & 83 or 84 or 85 or 86 or 87 or 88 or 89 or 90 or 91 \\
93 & 40 or 92 \\
94 & 69 and 82 and 93 \\
95 & Fabry.tw. \\
96 & Fabry's.tw. \\
97 & 95 or 96 \\
98 & Growth hormone.tw. \\
99 & 97 or 98 \\
100 & 94 not 99
\end{tabular}

\section{Data extraction}

A data extraction form will be developed to record and analyze information from each of the identified studies. The data will be extracted and verified by both reviewers in order to decrease reviewer error and bias. Data will be abstracted on study identification (authors, year of publication, and location of centers), characteristics of study design (sample size, inclusion and exclusion criteria, length of follow-up, type of hormone therapy, dosage of hormone therapy), characteristics of study participants (mean/median age, presence of comorbidities, stage of CKD, mean glomerular filtration rate (GFR), duration of CKD, time on dialysis), and outcome variable of interest (risk of all-cause mortality, cardiovascular mortality, and cardiovascular morbidity). Outcomes of RCTs and observational studies will be recorded separately. The data will be organized and managed in Endnote (version X7, Thomson Reuters, New York, NY) and Excel (version 2007, Microsoft Corporation, Redmond, WA).

\section{Risk of bias assessment}

Each study will be appraised for quality and risk of bias by each reviewer using the Cochrane risk of bias tool for randomized controlled trials for RCTs or the Cochrane risk of bias tool for cohort studies [37]. Additionally, a Jadad score will be calculated for each trial [38]. This will be used to determine the strength of the body of evidence.

\section{Data synthesis and analysis}

Data will be presented on the number and characteristic of articles identified for the initial screening and full-text review. The number of identified studies that were included and excluded (with reasons for exclusions) will be presented using the PRISMA flow chart [39].

The primary outcome of the risk ratio of all-cause mortality, cardiovascular mortality, and cardiovascular morbidity with hormone therapy compared to placebo or no hormone therapy will be reported from a potential metaanalysis of the RCTs and observational studies. If only the hazard ratio is presented, the number of events in each group will be abstracted from the study (if possible) and a risk ratio will be presented. If the above is not possible, we will present both risk ratio and hazard ratio estimates separately. The estimates will be presented separately for RCT and observational studies. As we anticipate the studies to be inherently different due to types of hormone therapy, dose regimen, etc., we will use the random effects model to pool estimates. Furthermore, statistical heterogeneity will be assessed using the Cochrane Q statistic and $I^{2}$ statistic. If there is significant statistical heterogeneity, univariate meta-regression analysis will be done to assess the effects of the following variables on risk estimates: mean age, diabetes, cardiac history, comorbidities, mean estimated glomerular filtration rate, CKD stage, and route of administration of hormone therapy. Evidence of publication bias will be determined through visual inspection of funnel plots and Begg's rank correlation test for asymmetry. All analysis will be performed using STATA (version 12, StataCorp LP, College Station, TX). If meta-analysis is not possible, we will present the results as a systematic review.

\section{Limitations}

Although this methodology had been designed to minimize bias, we anticipate some limitations with this review. First, we anticipate a difference in the formulation and dosage of hormone therapy in different studies. Restriction of the inclusion criteria to a specific formulation or dosage of hormone therapy would likely result in a paucity of data. Therefore, in order to control for any variations in outcomes due to differences in type and dose of hormone therapy in the included studies, we will perform subgroup analyses based on the type and doses of hormone therapy used to understand their contribution to heterogeneity.

Second, our results may be confounded by the lack of study-level data on the prevalence of important comorbidities such as diabetes and hypertension. Due to this, our results would have to be interpreted with caution; however, we will attempt to provide sufficient evidence to help determine the clinical significance of the presented pooled estimates by conducting stratified analyses when possible.

\section{Discussion}

In summary, this systematic review has the potential to have significant clinical implications. If a cardioprotective 
role of hormone therapy is identified, then this could provide a rationale for large multicenter RCTs to examine estrogen replacement as a therapy to mitigate the high $\mathrm{CV}$ risk in women with CKD. Conversely, if the cardioprotective effects of hormone therapy cannot be established, this evidence would be useful in enhancing both patients' and physicians' understanding of the limitations of this treatment.

To ensure that our findings have clinical impact on both patients and the physicians who care for them, we will disseminate our findings broadly by presenting at scientific conferences, publishing in a peer-reviewed journal as well as newsletters and websites of interested organizations, and conducting meetings with key stakeholders.

\section{Abbreviations}

CV: Cardiovascular; CVD: Cardiovascular disease; eGFR: Estimated glomerular filtration rate.

\section{Competing interests}

The authors declare that they have no competing interests.

\section{Authors' contributions}

SR conceived and designed the study, helped obtain funding for the study, registered the protocol with the PROSPERO database, and helped write the draft protocol. MCM, JMHL, SBW, MTJ, and EWS provided input into the design of the study and edited the draft protocol. SBA conceived and designed the study, obtained the funding, and helped write the draft protocol. All authors read and approved the final protocol.

\section{Acknowledgements}

SR is supported by the William Davies Scholarship and the Roy and Vi Baay Chair in Kidney Research Graduate Scholarship Award. MCM is supported by a ClHR doctoral scholarship. JHL. is funded as the Scientific Director of the Seniors Health Strategic Clinical Network within Alberta Health Services. SBW is supported by a grant from St. Jude Medical and Alberta Health Services. MTJ is supported by a KRESCENT new investigator award. EWS is supported by a National Institute of Health grant. SBA is supported by Alberta Innovates Health Solutions. Funding sources had no role in the study design, in the writing of the report, and in the decision to submit the article for publication. We thank Ms. Diane Lorenzetti and Ms. Helen Lee Robertson for conducting the literature searches and for peer reviewing the MEDLINE search strategy.

\begin{abstract}
Author details
${ }^{1}$ Faculty of Medicine, University of Calgary, 3330 Hospital Drive NW, Calgary T2N 4N1Alberta, Canada. ${ }^{2}$ Libin Cardiovascular Institute of Alberta, 140329 Street NW, Calgary T2N 2T9Alberta, Canada. ${ }^{3}$ Alberta Kidney Disease Network, 1403-29th St. NW, C210, Calgary T2N 2T9Alberta, Canada. ${ }^{4}$ Division of Endocrinology, Diabetes and Hypertension, Department of Medicine, Brigham and Women's Hospital, 75 Francis St., Boston, MA 02115, USA.
\end{abstract}

Received: 2 December 2014 Accepted: 25 February 2015

Published online: 08 April 2015

\section{References}

1. Arora P, Vasa P, Brenner D, Iglar K, McFarlane P, Morrison H, et al. Prevalence estimates of chronic kidney disease in Canada: results of a nationally representative survey. Can Med Assoc J. 2013;185:E417-23.

2. Go AS, Chertow GM, Fan D, McCulloch CE, Hsu C-y. Chronic kidney disease and the risks of death, cardiovascular events, and hospitalization. New England. J Med. 2004;351:1296-305.

3. Veldhuis JD, Johnson ML, Bolton WK. Analyzing pulsatile endocrine data in patients with chronic renal failure: a brief review of deconvolution techniques. Pediatric Nephrol. 1991;5:522-8.

4. Swamy AP, Woolf PD, Cestero RV. Hypothalamic-pituitary-ovarian axis in uremic women. J Lab Clin Med. 1979;93:1066-72.
5. Kannel WB, Hjortland MC, MCNamara PM, Gordon T. Menopause and risk of cardiovascular disease: the Framingham study. Ann Intern Med. 1976;85:447-52.

6. Podlutsky A, Ballabh P, Csiszar A. Oxidative stress and endothelial dysfunction in pulmonary arteries of aged rats. Am J Physiol Heart Circ Physiol. 2010;298:H346-51.

7. Ungvari Z, Labinskyy N, Gupte S, Chander PN, Edwards JG, Csiszar A. Dysregulation of mitochondrial biogenesis in vascular endothelial and smooth muscle cells of aged rats. Am J Physiol Heart Circ Physiol. 2008;294:H2121-8.

8. Kim KH, Bender JR. Membrane-initiated actions of estrogen on the endothelium. Mol Cell Endocrinol. 2009;308:3-8.

9. Brosnihan KB, Li P, Ganten D, Ferrario CM. Estrogen protects transgenic hypertensive rats by shifting the vasoconstrictor-vasodilator balance of RAS. Am J Physiol Regul Integr Comp Physiol. 1997;273:R1908-15.

10. Fewster ME, Pirrie RE, Turner DA. Effect of estradiol benzoate on lipid metabolism in the rat. Endocrinology. 1967;80:263-71.

11. Chambliss KL, Wu Q, Oltmann S, Konaniah ES, Umetani M, Korach KS, et al. Non-nuclear estrogen receptor alpha signaling promotes cardiovascular protection but not uterine or breast cancer growth in mice. J Clin Invest. 2010;120:2319-30.

12. Guetta V, Panza JA, Waclawiw MA, Cannon 3rd RO. Effect of combined 17 beta-estradiol and vitamin E on low-density lipoprotein oxidation in postmenopausal women. Am J Cardiol. 1995;75:1274-6.

13. Rosano GM, Vitale C, Marazzi G, Volterrani M. Menopause and cardiovascular disease: the evidence. Climacteric. 2007;10 Suppl 1:19-24.

14. Rossouw JE, Anderson GL, Prentice RL, LaCroix AZ, Kooperberg C, Stefanick $M L$, et al. Risks and benefits of estrogen plus progestin in healthy postmenopausal women: principal results From the Women's Health Initiative randomized controlled trial. JAMA. 2002;288:321-33.

15. Anderson GL, Limacher M, Assaf AR, Bassford T, Beresford SA, Black H, et al. Effects of conjugated equine estrogen in postmenopausal women with hysterectomy: the Women's Health Initiative randomized controlled trial. JAMA. 2004;291:1701-12.

16. Hlatky MA, Boothroyd D, Vittinghoff E, Sharp P, Whooley MA, Group HR. Quality-of-life and depressive symptoms in postmenopausal women after receiving hormone therapy: results from the Heart and Estrogen/Progestin Replacement Study (HERS) trial. JAMA. 2002;287:591-7.

17. Rossouw JE, Prentice RL, Manson JE, Wu L, Barad D, Barnabei VM, et al. Postmenopausal hormone therapy and risk of cardiovascular disease by age and years since menopause. JAMA. 2007;297:1465-77.

18. Modena MG, Sismondi P, Mueck AO, Kuttenn F, Lignieres B, Verhaeghe J, et al. New evidence regarding hormone replacement therapies is urgently required transdermal postmenopausal hormone therapy differs from oral hormone therapy in risks and benefits. Maturitas. 2005;52:1-10.

19. Grodstein F, Clarkson TB, Manson JE. Understanding the divergent data on postmenopausal hormone therapy. N Engl J Med. 2003;348:645-50.

20. Holley JL, Schmidt RJ. Changes in fertility and hormone replacement therapy in kidney disease. Adv Chronic Kidney Dis. 2013;20:240-5.

21. Wang GC, Zheng JH, Xu LG, Min ZL, Zhu YH, Qi J, et al. Measurements of serum pituitary-gonadal hormones and investigation of sexual and reproductive functions in kidney transplant recipients. Int J Nephrol. 2010;2010:612126.

22. Gomez F, de la Cueva R, Wauters JP, Lemarchand-Beraud T. Endocrine abnormalities in patients undergoing long-term hemodialysis. The role of prolactin. Am J Med. 1980;68:522-30.

23. Belchetz PE, Plant TM, Nakai Y, Keogh EJ, Knobil E. Hypophysial responses to continuous and intermittent delivery of hypopthalamic gonadotropin-releasing hormone. Science (New York, NY). 1978;202:631-3.

24. Hoyert DL, Xu J. Deaths: preliminary data for 2011. Natl Vital Stat Rep. 2012;61:1-51.

25. Carrero JJ, de Mutsert R, Axelsson J, Dekkers OM, Jager KJ, Boeschoten EW, et al. Sex differences in the impact of diabetes on mortality in chronic dialysis patients. Nephrol Dial Transplant. 2011;26:270-6.

26. Carrero JJ, de Jager DJ, Verduijn M, Ravani P, De Meester J, Heaf JG, et al. Cardiovascular and noncardiovascular mortality among men and women starting dialysis. Clin J Am Soc Nephrol. 2011;6:1722-30.

27. Villar E, Remontet L, Labeeuw M, Ecochard R. Effect of age, gender, and diabetes on excess death in end-stage renal failure. J Am Soc Nephrol. 2007;18:2125-34.

28. Ginsburg ES, Walsh B, Greenberg L, Price D, Chertow GM, Owen Jr WF. Effects of estrogen replacement therapy on the lipoprotein profile in postmenopausal women with ESRD. Kidney Int. 1998;54:1344-50. 
29. Park JS, Jung HH, Yang WS, Kim SB, Min WK, Chi HS. Effects of hormonal replacement therapy on lipid and haemostatic factors in post-menopausal ESRD patients. Nephrol Dial Transplant. 2000;15:1835-40.

30. Matuszkiewicz-Rowinska J, Skorzewska K, Radowicki S, Sokalski A, Przedlacki J, Niemczyk S, et al. The benefits of hormone replacement therapy in pre-menopausal women with oestrogen deficiency on haemodialysis. Nephrol Dial Transplant. 1999;14:1238-43.

31. Main C, Knight B, Moxham T, Gabriel Sanchez R, Sanchez Gomez LM, Roque i Figuls $\mathrm{M}$, et al. Hormone therapy for preventing cardiovascular disease in post-menopausal women. Cochrane Database Syst Rev. 2013;4:Cd002229.

32. Hodis HN, Mack WJ. Hormone replacement therapy and the association with coronary heart disease and overall mortality: clinical application of the timing hypothesis. J Steroid Biochem Mol Biol. 2014;142:68-75.

33. Shamseer $L$. Preferred reporting items for systematic reviews and meta-analyses for protocols (PRISMA-P). In: 21st Cochrane Colloquium. 2013

34. Higgins JP, Green S. Cochrane handbook for systematic reviews of interventions. Chichester: Wiley-Blackwell; 2008. Wiley Online Library; 2008.

35. PROSPERO Center for Reviews and Dissemination. National Institute of Health Research. http://www.crd.york.ac.uk/PROSPERO/display_record.asp?lD= CRD42014014566. Accessed 5 Mar 2015.

36. Sampson M, McGowan J, Cogo E, Grimshaw J, Moher D, Lefebvre C. An evidence-based practice guideline for the peer review of electronic search strategies. J Clin Epidemiol. 2009;62:944-52.

37. Likis FE, Andrews JC, Fonnesbeck CJ, Hartmann KE, Jerome RN, Potter SA, et al. Cochrane risk of bias tool. 2014.

38. Jadad AR, Moore RA, Carroll D, Jenkinson C, Reynolds DJM, Gavaghan DJ, et al. Assessing the quality of reports of randomized clinical trials: is blinding necessary? Control Clin Trials. 1996:17:1-12.

39. Moher D, Liberati A, Tetzlaff J, Altman DG. Preferred reporting items for systematic reviews and meta-analyses: the PRISMA statement. Ann Intern Med. 2009:151:264-9.

\section{Submit your next manuscript to BioMed Central and take full advantage of:}

- Convenient online submission

- Thorough peer review

- No space constraints or color figure charges

- Immediate publication on acceptance

- Inclusion in PubMed, CAS, Scopus and Google Scholar

- Research which is freely available for redistribution 